\title{
Estacionamiento Inteligente
}

\section{Smart Parking}

\author{
RODRÍGUEZ-MIRANDA, Gregorio †*, SANTOS-OSORIO, Rene, ORDAZ-BANDA, Carmen Sarahí \\ y LOPEZ-RIVERA, José Armando
}

Universidad Tecnológica de San Juan del Río

ID $1^{\mathrm{er}}$ Autor: Gregorio, Rodríguez-Miranda / ORC ID: 0000-0002-2512-892X, Researcher ID Thomson: S-5808-2018, CVU CONACYT ID: 246718

ID $1^{\mathrm{er}}$ Coautor: Rene, Santos-Osorio / ORC ID: 0000-0002-4411-7628, Researcher ID Thomson: G-3453-2019, CVU CONACYT ID: 619722

ID $2^{\text {do }}$ Coautor: Carmen Sarahí, Ordaz-Banda / ORC ID: 0000-0001-6728-9943

ID $3^{\text {er }}$ Coautor: José Armando, Lopez-Rivera / ORC ID: 0000-0002-3821-3058

DOI: $10.35429 /$ JOIE.2019.9.3.34.39

Recibido 16 de Febrero, 2019; Aceptado 31 de Marzo, 2019

\section{Resumen}

En este trabajo se presenta el desarrollo de un prototipo para el control de espacios de un estacionamiento, que, mediante una página web, monitoré y muestre en tiempo real los lugares disponibles. Para este proyecto se utiliza: una tarjeta Arduino Uno, sensores de proximidad LM393, cables macho/hembra, macho/macho, módulo wifi ESP8266 y dos LED; todos estos conectados entre sí por un enlace de comunicación desarrollado con código de Arduino, permitiendo transmitir y enviar datos en el serial de Arduino. Se realiza un enlace de comunicación del serial de Arduino con código en lenguaje java, funcionando como intermediario para la inserción de información en la base de datos. La aplicación web se desarrolló con el lenguaje de programación PHP y HTML y se conectó a la base de datos alojada en el servidor MySQL, utilizando como editor de código Sublime Text. Logrando así tener en la página web el monitoreo en tiempo real de los lugares disponibles del estacionamiento. Los resultados mostrados por el prototipo indican que probablemente sea factible la implementación de esta tecnología para hacer inteligentes los estacionamientos.

Estacionamiento inteligente, Serial de Arduino, Comunicación Arduino MySQL

\begin{abstract}
In this work it present the development of a prototype for the control of spaces in a parking lot, which through a web page monitors and displays in real time the available places, for this project it is used: an Arduino Uno card, proximity sensors Lm393, male / female, male / male cables, ESP8266 WIFI module and two LEDs; all these connected to each other by a communication link developed with Arduino code, allowing to transmit and send data in the Arduino serial. An Arduino serial communication link is made with java language code, acting as an intermediary for the insertion of information in the database. The web application was developed with the programming language PHP and HTML and was connected to the database hosted on the MySQL server, using as a Sublime Text code editor. Thus achieving on the web page the monitoring in real time of the available parking places. The results shown by the prototype indicate that it is probably feasible to implement this technology to make the parking lots smart.
\end{abstract}

Smart parking, Arduino Serial, Arduino MySQL communication

Citación: RODRÍGUEZ-MIRANDA, Gregorio, SANTOS-OSORIO, Rene, ORDAZ-BANDA, Carmen Sarahí y LOPEZRIVERA, José Armando. Estacionamiento Inteligente. Revista de Ingeniería Innovativa. 2019. 3-9: 34-39

\footnotetext{
*Correspondencia al Autor (Correo Electrónico: grodriguezm@utsjr.edu.mx)

$\dagger$ Investigador contribuyendo como primer autor.
} 


\section{Introducción}

Un estacionamiento inteligente o smart parking se pude definir como el uso de tecnologías avanzadas para la operación eficiente, el monitoreo y la administración del estacionamiento dentro de una estrategia de movilidad urbana. (Departament of Mechatronics, Heliopolis University)

En muchas ciudades, el encontrar dónde estacionarse se ha convertido en un problema de movilidad, tránsito vehicular, contaminación, gasto de combustible, espacio, pérdida de tiempo y dinero. Un buen ejemplo de una urbe con esta problemática es la Ciudad de México, en esta megalópolis se registra un parque vehicular de cerca de 6 millones de automóviles según el Instituto Nacional de Estadística y Geografía (INEGI).

La Ciudad de México ocupa el nada honroso primer lugar con mayor tráfico vehicular en el mundo generando 59 minutos extras de tiempo en viajes por día consigna el TomTom Traffic Index.

El 30\% del tráfico generado se debe a que los conductores tardan entre 15 y 20 minutos en encontrar un lugar donde estacionar su auto, según datos revelados por el Instituto de Políticas para el Transporte y el Desarrollo (ITDP). En el estado de Querétaro en el 2017, según INEGI, se registraron 508,206 nuevos vehículos que circulan en nuestras ciudades, por lo que los usuarios tienen problemas para encontrar un lugar de estacionamiento en las horas pico.

El problema del estacionamiento en las ciudades, el cuidado del medio ambiente y la búsqueda de rentabilidad en cuanto a movilidad, hace que globalmente las empresas estén buscando soluciones basadas en las necesidades de cada ciudad.

En este sentido, la creación de estacionamientos inteligentes o smart parkings, desarrollando soluciones en base a tecnologías de bajo costo como, Arduino, utilizando lenguajes de programación de código abierto, como java, PHP, bases de datos como MySQL server y empleando metodologías ágiles como SCRUM, puede ser una alternativa que contribuya a resolver esta problemática.
En los últimos años el crecimiento de Internet de las cosas (IoT) por sus siglas en Ingles, a permitido el uso de nuevas tecnologías en la solución de diferentes problemas. La visión principal de Internet de las cosas (IoT) es la creación de un mundo inteligente donde lo real, lo digital y lo virtual convergen para crear un entorno que proporcione más inteligencia a la energía, la salud, el transporte, las ciudades, la industria, los edificios y muchas otras áreas de la vida diaria.

\section{Objetivo}

El presente trabajo tiene como objetivo, proporcionar una herramienta que permita a los usuarios de vehículos, que necesiten localizar un espacio de estacionamiento, lograrlo en el menor tiempo posible y lo más cerca de su destino, con esto se pretende disminuir el tránsito vehicular, la contaminación y los gastos de combustible. Otro factor importante en el que los usuarios se beneficiarían, sería el sentirse mejor al optimizar su tiempo de busqueda de estacionamiento, teniendo oportunidad de pasar mayor tiempo con su familia u otras actividades importantes para ellos.

\section{Metodología}

Para el desarrollo del software, decidimos utilizar la metodología Scrum, ya que es un marco de trabajo simple que promueve la colaboración en los equipos, para lograr desarrollar productos complejos.

Scrum es un marco de trabajo para desarrollo ágil de software. Adopta una estrategia de desarrollo incremental, en lugar de la planificación y ejecución completa del producto.

Scrum es un proceso de gestión que reduce la complejidad en el desarrollo de productos para satisfacer las necesidades de los clientes. La gerencia y los equipos de Scrum trabajan juntos alrededor de requisitos y tecnologías, para entregar productos funcionando de manera incremental. En la contrucción del prototipo elaboramos una maqueta con material de cartón para la contrucción de los espacios de estacionamiento, utilizamos ua tarjeta protoboard y cables machomacho, macho-hembra y hembra-hembra para las conecciones con los sensores y los leds. 
Se programó la tarjeta Arduino Uno utilizando su interface nativa en lenguage micro c, para recibir la señal de los sensores de proximidad LM393, instalados en cada espacio de estacionamiento, dependiendo de la señal recibida el programa envía la orden a los actuadores para indicar que el espacio de estacionamiento esta ocupado o libre, para esta función se utilizan LED de color verde y rojo, para indicar espacio libre o espacio ocupado respectivamente.

El módulo WIFI ESP8266 envía los datos a la aplicación web, para que esta almacene la información de espacios libres y ocupados en la base de datos MySQL. El usuario del estacionamiento puede entrar a la página web a consultar la información del estacionamiento en tiempo real, para saber donde hay espacios libres para estacionarse.

\section{Marco Teórico}

De acuerdo a la página oficial de la placa base Arduino es: "Una plataforma electrónica de código abierto basada en hardware y software fáciles de usar. Las placas Arduino pueden leer entradas (luz en un sensor, un dedo en un botón o un mensaje de Twitter) y convertirla en una salida: activar un motor, encender un LED y publicar algo en línea.

Puede decirle a su tarjeta qué debe hacer enviando un conjunto de instrucciones al microcontrolador de la tarjeta. Para hacerlo, utiliza el lenguaje de programación Arduino (basado en Wiring) y el software Arduino (IDE), basado en el procesamiento.

A lo largo de los años, Arduino ha sido el cerebro de miles de proyectos, desde objetos cotidianos hasta instrumentos científicos complejos. Una comunidad mundial de creadores (estudiantes, aficionados, artistas, programadores y profesionales) se han reunido en torno a esta plataforma de código abierto, sus contribuciones han sumado una cantidad increíble de conocimientos accesibles que pueden ser de gran ayuda para principiantes y expertos.

El Internet de las cosas (IoT) es la nueva estructura que, según las predicciones, en el 2020 conectará 50,000 millones de objetos inteligentes cuando la población mundial alcance los 7,600 millones.

\section{Como sugiere la ITU (Unión} Internacional de Telecomunicaciones), esta estructura fundamental se construirá alrededor de una arquitectura multicapas en la cual los objetos inteligentes se usarán para prestar diferentes servicios a través de las cuatro capas principales [Liñán Colina et al., 2016], una capa del dispositivo, una capa de red, una capa de soporte y una de aplicación.

La capa del dispositivo contiene los sensores, actuadores, dispositivos RFID y pasarelas "gateways" que se usan para recolectar las lecturas del sensor para su procesamiento posterior, mientras que la capa de red proporciona el transporte necesario y las capacidades de red para enrutar los datos de IoT a los sitios de procesamiento. La capa de soporte es una capa intermedia (middleware) que sirve para esconder la complejidad de las capas inferiores a la capa de aplicación y para dar servicios específicos y genéricos tales como almacenamiento bajo formas diferentes (sistemas de manejo de base de datos y/o sistemas de computación en la nube), así como otros servicios, por ejemplo, traducción.

Los avances tecnológicos han permitido el desarrollo de circuitos integrados de muy pequeño tamaño, alta eficiencia energética y bajo costo. Existen diversas implementaciones de Estacionamientos inteligentes en el mundo [Vera-Gómez et al., 2016; Khanna \& Anand, 2016] y el área se encuentra en constante investigación. El estudio principal está basado en la mejora de los dispositivos de detección de espacios libres, p. ej. Baroffio et al. [2015] propone un dispositivo específico además de existir patentes al respecto como "Apparatus and method for sensing the occupancy status of parking spaces in a parking lot" [Winter \& Osterweil, 2006]. Dentro de las áreas de estudio está el interés por la optimización y subida a la nube de los datos de los espacios ocupados [Adki \& Agarkhed, 2016] y procesamiento de big data producido por los mismos y por el tráfico de la ciudad medido con sensores [Nandury \& Begum, 2016], minimización de cruces de vehículos en el estacionamiento [Tsiaras et al., 2015], optimizaciones para reducir las emisiones de gases que generan el efecto invernadero [Ramaswamy, 2016; Sagar et al., 2016] incluyendo mejoras en la disposición de los vehículos eléctricos para optimizar la carga de los mismos [Kuran, 2015]. 
Todo esto en base a lo descrito en el estudio "Estacionamiento Inteligente con IoT" [Enrique Calot et al., 2017] de la Universidad Nacional de La Plata, Facultad de Informática. Uno de los objetivos del presente trabajo es el uso de tecnología de bajo costo para lograr resultados similares a los descritos, como el sensor de proximidad LM393.

\section{Diseño y Tecnología propuestos}

Los estacionamientos públicos, de grandes empresas, supermercados, aeropuertos y plazas comerciales, en su mayoría, no cuentan con un sistema inteligente de bajo costo, que reduzca el tiempo de búsqueda de lugares libres y optimice la tarea de estacionarse, guiando al usuario de acuerdo a su ubicación y destino. El presente proyecto, se realizó utilizando tecnologías de Internet de las cosas con dispositivos de bajo costo, empleando protocolos de comunicación wifi, frameworks de programación y base de datos de código libre.

El IoT integrado a la tecnología movil es una tecnología disponible para la solución de los problemas que se presentan en las ciudades como el de estacionamiento inteligente.

Se construyó un prototipo de estacionamiento, con dos espacios de estacionamiento, se instalaron dos LEDs en cada espacio, uno rojo para indicar que está ocupado y uno verde para indicar que esta libre. Ademas se instalaron dos sensores de proximidad, uno en el techo (estructura superior) y uno en la parte de atrás (pared o estructura), los sensores están conectados a la tarjeta Arduino UNO, como la tarjetas arduino tienen un límite de puertos, 14 digitales y 6 analógicos, no es práctica para un proyecto real con decenas o cientos de espacios de estacionamiento, para ese caso se debe configurar con tarjetas que cuenten con un número mayor de puertos o bien hacer arreglos con las tarjetas Arduino y un servidor que multiplexe las señales para tener la capacidad de procesamiento requerida.

La tarjeta Arduino UNO se configuró con un módulo WIFI para enviar la señal a la aplicación web, despues de procesar la señal se almacena la información en la base de datos MySQL.
Se actualiza el estatus de cada uno de los espacios que están enviando señal, ya sea cambiar de ocupado a libre o viceversa, para que pueda ser consultada por cualquier usuario desde su dispositivo movil o computadora y pueda encontrar un espacio de estacionamiento libre con más certidumbre y prontitud, evitando congestionar el tráfico, ahorrando combustible y contaminando menos al medio ambiente.

\section{Resultados Obtenidos}

El proyecto de estacionamiento inteligente está diseñado considerando principalmente la calidad y la asertividad. Para probar estas dos características, desarrollamos un prototipo para evaluar su fiabilidad. El área de prueba consistió en dos espacios de estacionamiento, donde se instalaron dos sensores de proximidad LM393 y dos LEDs, un verde y un rojo, en cada espacio, conectados a una tarjeta Arduino UNO, la tarjeta se conectó al módulo de comunicación WIFI, también se utilizaron dos vehiculos miniatura para la prueba.

El sensor LM393, tiene un margen de detección entre 2 a 3 centimetros, por esta razón se instalaron dos para cada espacio de estacionamiento, uno en la parte posterior baja y otro en la parte superior al frente, con la finalidad de que al menos uno de los dos registre el evento de ingreso y/o de egreso del vehículo.

Las observaciones de prueba se centran en dos estados del lugar de estacionamiento:

\section{a. Hay un vehículo en el lugar}

b. No hay vehículo en el lugar

Cuando la aplicación detecta que un sensor está enviado información de proximidad de un objeto, evento a), entonces la aplicación actualliza el estado de la base de datos, poniendo ocupado un lugar más de estacionamiento, envía la señal al módulo WIFI para que indique a la tarjeta arduino apagar el LED verde y encender el rojo, en este momento los usuarios de la aplicación web verán, en la página del estacionamiento, que hay un lugar menos disponible. 
Cuando la aplicación detecta que los dos sensores están enviando información que no hay objetos en su rango de proximidad, entonces la aplicación actualiza la base de datos, agregando un espacio de estacionamiento más a los espacios libres, además envía al módulo WIFI la orden para que le indique a la tarjeta Arduino apagar el LED rojo y encender el verde, desde este momento los usuarios de la aplicación verán actualizados los lugares de estacionamiento disponibles.

Únicamente cuando los dos sensores no detectan presencia, entonces el led verde prende (On) y el led rojo se apaga (Off), indicando espacio de estacionamiento libre, en cualquier otro caso el led verde se apaga y el led rojo se prende, indicando espacio de estacionamiento ocupado.

\begin{tabular}{|l|l|l|l|}
\hline $\begin{array}{c}\text { Sensor superior } \\
\text { frontal }\end{array}$ & \multicolumn{1}{c}{$\begin{array}{c}\text { Sensor posterior } \\
\text { bajo }\end{array}$} & $\begin{array}{c}\text { Led } \\
\text { rojo }\end{array}$ & $\begin{array}{c}\text { Led } \\
\text { verde }\end{array}$ \\
\hline Presencia & Presencia & On & Off \\
\hline Presencia & Ausente & On & Off \\
\hline Ausente & Presencia & On & Off \\
\hline Ausente & Ausente & Off & On \\
\hline
\end{tabular}

Tabla 1 Estados de los LEDs

Fuente: Elaboración Propia

Despues de haber realizado cincuenta pruebas de estacionar un vehiculo y cincuenta pruebas de retirar un vehiculo en los espacios de estacionamiento, se observo que en el 92 por ciento de los intentos el sistema funciono perfectamente, teniendo un 8 por ciento de oportunidad de mejora.

\section{Conclusiones}

Al terminar el proyecto, podemos concluir que el utilizar tecnología barata en la contrucción de un estacionamiento inteligente apoya la parte económica, permitiendo la experimentación en este terreno, sin embargo observamos que para su implementación en proyectos reales puede quedar corta en prestaciones y limitada en alcance.

Un factor importnte es que el prototipo funciona bien, con lo que podemos inferir que los objetivos esperados de disminuir el tráfico, disminuir el consumo de combustible, acortar el tiempo en encontrar un espacio de estacionamiento y contribuir a disminuir la contaminación del medio ambiente, se pueden lograr en un ambiento de producción real.
Consideramos que la investigación del presente proyecto apoya el avance científico del area en el sentido de que brindamos una visión diferente, sobre todo en la parte económica, de abordar esta problemática, además de ampliar las investigaciones y avances sobre el tema.

\section{Proyección a Futuro}

En base a las estadísticas que muestra el INEGI, podemos afirmar que la demanda de espacios de estacionamiento en los próximos años seguirá en incremento.

Cada día existen más usuarios que requieren sistemas de estacionamiento inteligente y aunque ya existen en el mercado varias opciones, los objetivos del presente prototipo son: brindar un servicio de calidad a bajo costo, utilizando las nuevas tecnoloígas de IoT ya que cada día se han hecho más accesibles y con mayores prestaciones, ademas de presentar una interface amigable con facilidad de uso que apoye su adopsión en el mercado. Como objetivos secundarios, pero no menos importantes, este proyecto apoya en la reducción de emisiones contaminantes al encontrar más rápido un espacio de estacionamiento, reduciendo las congestiones de tráfico, además permite a los usuarios gastar menos tiempo para llegar a sus destinos, ahorrando dinero en combustible, apoyando a mejorar el medio ambiente.

Para la recepción de las señales de los sensores y el envio de ordenes a los actuadores (LEDs) se utilizó la tarjeta Arduino Uno y un módulo WIFI, es probable que para estacionamientos grandes esta tecnología no sea suficiente, así que podría utilizarse alguna otra tarjeta o computadora de hardware reducido como Raspberry $\mathrm{Pi}$, que también son económicas y que brindan mayores prestaciones que la Arduino Uno, como por ejemplo, ya cuenta con comunicación WIFI integrada, tiene un puerto general de entrada/salida, GPIO por sus siglas en inglés, de 40 pines, puerto Ethernet, puertos USB, etc. Otro elemento que tiene oportunidades de mejora es el sensor LM393, por la restricción de distancia, se podría usar un sensor ultrasonico que tiene mucho mayor alcance. 
Un elemento muy importante en este tipo de sistemas es la asistencia al usuario para la localización del espacio libre de estacionamiento por lo que podrían agregarse camaras y pantallas en los accesos y pasillos del estacionamiento para detectar donde está el vehículo e indicarle en las pantallas la ruta a seguir, además de estos dispositivos, se requiere que la aplicación reconozca el vehiculo por medio de alguna tecnología como la lectura de las placas o la lectura de un codigo QR que podría generarse y pegarse al parabrisas del vehículo en el lugar de acceso al estacionamiento.

\section{Referencias}

Adeel Javed (2016), Building Arduino Projects for the Internet of Things: Experiments with Real-World Applications

Calot Enrique, Maluf Mariano, Neffa Marcelo. (2017). Estacionamiento Inteligente con IoT Universidad Nacional de La Plata, Facultad de Informática

Galeano, S. L. G., \& Franco, Á. S. O. (2019). Viabilidad de aplicación móvil para la búsqueda de plazas de estacionamientos libres en el Centro de Negocios de Asunción. Revista ScientiAmericana, 6(1), 17-21.

GSMA (2017). Smart Parking: A Guide to Ensuring a Successful Mobile IoT Deployment www.gsma.com/smartcities

\section{INEGI}

https://www.inegi.org.mx/sistemas/olap/consult a/general_ver4/MDXQueryDatos.asp?\#Regreso $\& c=$

Ken Schwaber, Jeff Sutherland (2012) Editorial Wiley $\mathrm{N}^{\circ}$ páginas 216 Isbn 1118206665

Parwekar, P. (2011). From Internet of Things towards cloud of things. In Computer and Communication Technology (ICCCT), 2nd International Conference on (pp. 329-333). IEEE

Sagar, S.V., Balakiruthiga, B., \& Kumar, A.S. (2016). Novel vehicle booking system using IOT. In Green Engineering and Technologies (IC-GET), Online International Conference on (pp. 1-5). IEEE.
The home of the scrum (2019) https://www.scrum.org/resources/blog/que-esscrum

V. Kepuska, Humaid Alshamsi (2016), Article Smart Car Parking System https://www.researchgate.net/publication/31934 5084 